BMJ Open Diabetes Research \& Care

\section{Gender-related affecting factors of prediabetes on its 10-year outcome}

To cite: Song $X$, Qiu M, Zhang $\mathrm{X}$, et al. Gender-related affecting factors of prediabetes on its 10-year outcome. BMJ Open Diabetes Research and Care 2016;4: e000169. doi:10.1136/ bmjdrc-2015-000169

$\mathrm{XS}, \mathrm{MQ}$, and $\mathrm{XZ}$ contributed equally.

Received 9 November 2015 Revised 12 February 2016 Accepted 4 March 2016

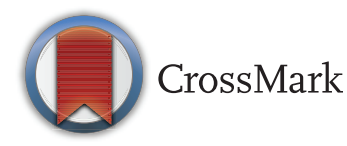

${ }^{1}$ Department of

Endocrinology and Metabolism, Shanghai Clinical Center for Endocrine and Metabolic Diseases, Shanghai Institute of Endocrine and Metabolic Diseases, RuiJin Hospital, Shanghai Jiao Tong University School of Medicine, Shanghai, China ${ }^{2}$ Department of Endocrinology and Metabolism, Yangpu Hospital, Tongji University School of Medicine, Shanghai, China

${ }^{3}$ Ping Liang Community Health Service Center, Yang Pu District, Shanghai, China ${ }^{4}$ Department of Diabetes and Metabolic Diseases Research, Beckman Research Institute, City of Hope National Medical Center, Duarte, CA, USA

Correspondence to Dr Jingyan Tian; tianjypaper@163.com Weiqing Wang; wqingw61@163.com

\section{ABSTRACT}

Objective: To investigate the gender-related affecting factors of prediabetes on its 10-year outcome, in a longitudinal study.

Methods and results: This longitudinal populationbased study was performed in the Ping Liang community, Yangpu district, Shanghai, between November 2002 and October 2014. There were 334 participants with prediabetes enrolled in the final analysis. While a certain proportion of the prediabetic population progress to diabetes, the majority remain at the same level or even revert to normal glucose regulation. No gender difference was observed in the change of glucose regulation. However, results from an adjusted logistic regression analysis in males showed that physical activity was significantly associated with both elevated odds of reverting to normal glucose regulation (active vs inactive, OR $3.00,95 \% \mathrm{Cl} 1.09$ to 8.30) and developing diabetes (OR $0.34,95 \% \mathrm{Cl} 0.13$ to 0.92 ). Age, baseline $2 \mathrm{~h}$ glucose, triglycerides and smoking status were also risk factors significantly associated with diabetes development; while for females, waist circumference played a key role in the outcome. Every unit elevation of waist circumference was associated with lower odds of reverting to normal glucose regulation (OR, $0.94 ; 95 \% \mathrm{Cl} 0.89$ to 0.98$)$ and higher odds of progressing to diabetes (OR, 1.05; $95 \%$ $\mathrm{Cl} 1.01$ to 1.10). Baseline hypertension and family history of diabetes carried higher risk for developing diabetes as well.

Conclusions: Physical activity in males and waist circumference in females are important factors predicting both progression to diabetes and regression to normal glucose regulation, indicating that more exercise for males and lower waist circumference for females are beneficial for prediabetes to achieve reversion.

\section{INTRODUCTION}

Prediabetes, which presents before diabetes, has been increasing globally, and the number of people with prediabetes worldwide is estimated to reach 472 million by the year $2025 .{ }^{1}$ In 2010 , the prevalence of prediabetes was estimated to reach $36.2 \%$ in the $\mathrm{USA}^{2}$ and $50.1 \%$ in China. ${ }^{3}$

A number of clinical studies have focused on diabetes prevention in people with prediabetes. $^{4-6}$ These studies have showed $25-$

\section{Key messages}

- Part of the prediabetes population reverted to normal after 10 years.

- Physical activity is important in the outcome of prediabetes in males.

- Waist circumference is important in the outcome of prediabetes in females.

$67 \%$ reductions in the incidence of diabetes over 2.5-6 years of intervention, with most participants remaining in a prediabetic state. However, while many trials ${ }^{4-8}$ have demonstrated the effectiveness of lifestyle and/or drug therapy in preventing diabetes in people with prediabetes, only a few have examined the effect of an intervention on returning the prediabetes state to normal glucose regulation. ${ }^{4} 78$ Moreover, it was reported that risk factors for diabetes differed by gender, therefore gender-specific care was recommended for patients with diabetes. $^{9}{ }^{10}$ However, no previous study was found about gender-related risk factors and treatment for reverting prediabetes to normal glucose regulation. In fact, even if diabetes could be delayed or prevented, both microvascular and macrovascular disease appear more prevalent in those with prediabetes compared with their normoglycemic peers. ${ }^{11}$ Hence, there is growing consensus that normoglycemia should be the treatment goal for people with prediabetes.

Although risk factors of developing diabetes are well established, ${ }^{12}$ far less is known about factors affecting the transformation from prediabetes to normal glucose regulation. ${ }^{13}$ There is a variety of possible contributors, including genetic factors, environmental exposures, physical activity, and metabolic disorders. A long-time study may provide a chance to explore some of these possible mediators; we performed a 10-year follow-up study to investigate the gender-related outcome of prediabetes and basal biological factors on the incidence of progression to diabetes, and regression to normal glucose regulation. 


\section{RESEARCH DESIGN AND METHODS}

\section{Study population}

This study was from a population-based prospective cohort study of 2132 men and women aged 18-76 years, from November 2002 to January 2003, among whom 778 participants were prediabetic at baseline. The study design has been described previously. ${ }^{14}{ }^{15}$ Briefly, a sample of 2200 people were randomly selected from 18000 eligible residents and valid information was obtained from 2132 people in the sample. This follow-up visit was conducted from July 2013 to October 2014; $526(67.7 \%)$ participants who were prediabetic at baseline were followed, among whom 153 answered questionnaires only and had no available glycemic data, and 39 participants died. The remaining 334 individuals were included in our final analysis (figure 1).

The study protocol was approved by the institutional review board of our hospital. Written informed consent was obtained from all participants before data collection.

\section{Data collection}

A standard questionnaire was administered by trained staff to acquire information on demographic characteristics, personal and family medical history, lifestyle including dietary habits, physical activity, smoking and drinking habits, etc. Physical activity level at leisure time was calculated as the product of the duration and frequency of each activity (in hours per day) weighted by an estimate of the metabolic equivalent (MET) of that activity. ${ }^{16}$ We defined the lowest tertile of METs as inactive and the upper two tertiles as active. Waist circumference was measured at the narrowest point below the ribs or halfway between the lowest ribs and the iliac crest in centimeters. Assessment methods of other variables have been specifically described elsewhere. ${ }^{14} 15$

\section{Measurement of blood glucose}

Venous blood samples were drawn at baseline and follow-up. The blood specimen analysis in the follow-up

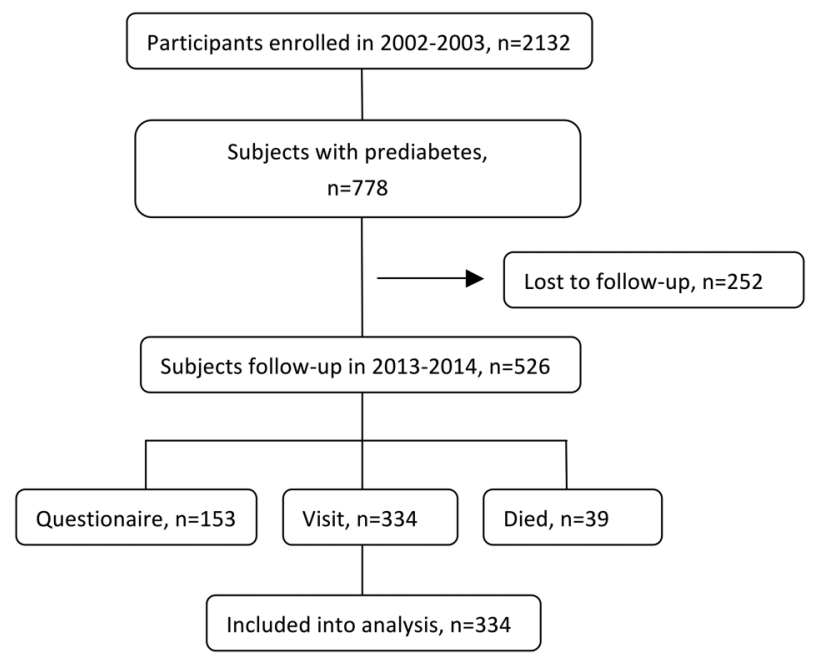

Figure 1 Details on the studied population. visit was performed in the same laboratory, using the same method as in the initial visit. ${ }^{14}$ Blood for fasting blood glucose was drawn between 06:30 and 09:30 after an overnight fast. We used a $75 \mathrm{~g}$ liquid glucose load to assess $2 \mathrm{~h}$ glucose for those without previously known diabetes, and $100 \mathrm{~g}$ steamed bread that contained approximately similar carbohydrates for those with selfreported diabetes at baseline. Owing to the steamed bread test showing extra benefits in keeping sensitive individuals from adverse effects such as nausea, vomiting, and wild fluctuations of glucose, $100 \mathrm{~g}$ steamed bread was used for all participants at follow-up. These two modes of assessment of glucose tolerance proved to be of equal clinical significance for diabetes diagnosis and of equal effectiveness in evaluating residual $\beta$ cell function in normal glucose regulated as well as in diabetic participants. ${ }^{17-19}$ Blood samples were centrifuged to separate plasma and analyzed immediately after collection. Glucose levels were assessed using glucose oxidase methods, glycated hemoglobin (HbAlc) was tested using high performance liquid chromatography (HPLC) methods, and triglyceride and cholesterol levels were measured using enzymatic methods.

\section{Outcome definitions}

Prediabetes was defined as fasting glucose between 5.6 and $6.9 \mathrm{mmol} / \mathrm{L}$ and/or $2 \mathrm{~h}$ glucose between 7.8 and $11.0 \mathrm{mmol} / \mathrm{L}$ at baseline, while $\mathrm{HbAlc}$ between $5.7 \%$ and $6.4 \%$ was added to the diagnostic standard at follow-up according to the American Diabetes Association (ADA) suggestion from 2010. Diabetes was decided either by $2 \mathrm{~h}$ glucose $\geq 11.1 \mathrm{mmol} / \mathrm{L}$ and/or fasting glucose $\geq 7.0 \mathrm{mmol} / \mathrm{L}$, or self-reported diabetes at baseline. HbAlc $\geq 6.5 \%$ was an additional standard as well at follow-up. ${ }^{20}$

\section{Statistical analyses}

Baseline demographic and metabolic characteristics were described in means $( \pm \mathrm{SD})$ for continuous variables and $\mathrm{n}(\%)$ for categorical variables. We analyzed the significance of differences between groups by gender difference using Student's t test for continuous variables and the $\chi^{2}$ test for categorical variables. Student's t test was applied to analyses for comparisons of basic characteristics of the follow-up population with those who were lost to follow-up. Binary logistic regression models were used to examine the association of metabolic factors with the odds of the main outcomes (progressed to overt diabetes or regressed to normal glucose regulation). The analyses were performed separately in males and females, adjusting for age, family history of diabetes, hypertension status, waist circumference, physical activity, glycemic and lipid levels, and smoking and drinking status at baseline. An $\mathrm{OR}>1$ indicates greater risk for regression (ie, favors regression), whereas the opposite is true for $\mathrm{OR}<1$ (ie, impedes regression). All statistical tests were two-sided, and a $p$ value of $<0.05$ was considered statistically significant. 
All statistical analyses were conducted with SPSS for Windows, V.18.0 (SPSS, Chicago, Illinois, USA).

\section{RESULTS}

The follow-up duration was 10.5-12.0 years (mean 10.8 years). The first examination was conducted from November 2002 to January 2003. The follow-up visit lasted from July to December 2013, but an additional follow-up was carried out from September to October 2014, for those unavailable at the earlier visit. General characteristics of the study population are presented in table 1 according to gender difference. In general, males tended to have a higher consumption level of cigarettes and alcohol, and higher tertiary education level, while females were more likely to have adverse lipid profiles. There was no significant difference in age, blood glucose level, body mass index (BMI), hypertension rate, family history of diabetes, and physical activity, between males and females. Meanwhile, we compared the prediabetic participants who attended the follow-up with those who were lost to follow-up, and found no significant difference in any baseline characteristics (data not shown).

Table 2 demonstrates the 10-year outcome of prediabetes. During the visit, the overall incidences of diabetes were $98(29.3 \%)$; $161(48.2 \%)$ participants remained prediabetes after 10 years. Interestingly, $75(22.5 \%)$ participants with prediabetes did not progress but returned to normal glucose regulation. No gender difference was observed in the change of glucose regulation, with incidences of restoration of $22.4 \%$ in males and $22.5 \%$ in females, and incidences of progression being $29.6 \%$ in males and $29.2 \%$ in females.

Table 3 shows the ORs for reverting to normal glucose regulation by gender-related risk factors. Generally speaking, after adjusting for age, family history of diabetes, waist circumference, blood glucose and lipid levels, smoking and drinking status, physical activity, and hypertension status at baseline, there were age and physical activity in males and blood glucose level and waist circumference in females, which were significantly associated with the odds of reverting to normal glucose regulation, respectively. Active physical activity in males had an OR of 3.00 and a $95 \%$ CI (1.09 to 8.30) for regression compared with inactive participants. Every unit elevation of waist circumference in females was associated with an OR and a $95 \%$ CI of $0.94,95 \%$ CI (0.89 to 0.98) for reverting back to normal glucose regulation.

Table 4 shows the ORs for developing diabetes by gender-related risk factors (adjusted for the same covariates as in table 3). The odds of progressing into diabetes increased remarkably in male participants with older

Table 1 General characteristics in males and females at baseline

\begin{tabular}{|c|c|c|c|c|}
\hline Value & Overall $(n=334)$ & Male $(n=125)$ & Female $(n=209)$ & p For difference \\
\hline Age (year) & $57.2 \pm 10.0$ & $57.2 \pm 11.0$ & $57.2 \pm 9.4$ & 0.96 \\
\hline Fasting glucose (mmol/L) & $6.0 \pm 0.4$ & $6.0 \pm 0.4$ & $6.0 \pm 0.4$ & 0.95 \\
\hline $2 \mathrm{~h}$ glucose $(\mathrm{mmol} / \mathrm{L})$ & $5.9 \pm 1.6$ & $6.0 \pm 1.8$ & $5.9 \pm 1.4$ & 0.70 \\
\hline LDL-c (mmol/L) & $2.96 \pm 0.76$ & $2.84 \pm 0.74$ & $3.03 \pm 0.77$ & 0.02 \\
\hline HDL-c (mmol/l) & $1.40 \pm 0.34$ & $1.27 \pm 0.31$ & $1.48 \pm 0.34$ & $<0.001$ \\
\hline Total cholesterol (mmol/L) & $5.09 \pm 0.94$ & $4.89 \pm 0.94$ & $5.21 \pm 0.92$ & 0.002 \\
\hline Triglycerides $(\mathrm{mmol} / \mathrm{L})^{\star}$ & $1.42(0.99-1.93)$ & $1.56(1.15-2.19)$ & $1.33(0.93-1.84)$ & 0.005 \\
\hline Waist circumference $(\mathrm{cm})$ & $82.2 \pm 9.1$ & $85.9 \pm 8.9$ & $80.1 \pm 8.5$ & $<0.001$ \\
\hline Systolic blood pressure (mm Hg) & $134.4 \pm 19.6$ & $136.0 \pm 19.8$ & $133.4 \pm 19.4$ & 0.25 \\
\hline Diastolic blood pressure $(\mathrm{mm} \mathrm{Hg})$ & $84.6 \pm 11.5$ & $86.4 \pm 12.1$ & $83.6 \pm 11.0$ & 0.03 \\
\hline Physical activity (\%) & & & & 0.41 \\
\hline Inactive & 36.8 & 40.0 & 34.9 & \\
\hline Active & 63.2 & 60.0 & 65.1 & \\
\hline Alcohol use (\%) & & & & $<0.001$ \\
\hline Currently & 14.1 & 30.4 & 4.3 & \\
\hline Formerly & 2.7 & 6.4 & 0.5 & \\
\hline Never & 83.2 & 63.2 & 95.2 & \\
\hline Smoking status (\%) & & & & $<0.001$ \\
\hline Current smoker & 18.9 & 46.4 & 2.4 & \\
\hline Former smoker & 4.5 & 12.0 & 0 & \\
\hline Never smoked & 76.6 & 41.6 & 97.6 & \\
\hline Educational background (\%) & & & & $<0.001$ \\
\hline Primary education & 59.3 & 45.6 & 67.5 & \\
\hline Secondary education & 27.2 & 24.8 & 28.7 & \\
\hline Tertiary education & 13.5 & 29.6 & 3.8 & \\
\hline
\end{tabular}

Results are given mean \pm SD or $n(\%)$.

*Values of fasting triglycerides did not follow Gaussian distribution, thus medium and interquartile ranges were used instead of mean and SD to describe central and discrete tendency.

HDL-c, high-density lipoprotein cholesterol; LDL-c, low-density lipoprotein cholesterol. 
Table 2 Gender-related 10-year outcome of prediabetes

\begin{tabular}{|c|c|c|c|c|}
\hline \multirow[b]{2}{*}{ Gender } & \multirow[b]{2}{*}{$\begin{array}{l}\text { Case } \\
\text { (n) }\end{array}$} & \multicolumn{3}{|c|}{ Outcome of 10 -year follow-up n (\%) } \\
\hline & & $\begin{array}{l}\text { Normal glucose } \\
\text { regulation }\end{array}$ & Prediabetes & Diabetes \\
\hline Male & 125 & $28(22.4)$ & $60(48.0)$ & 37 (29.6) \\
\hline Female & 209 & $47(22.5)$ & 101 (48.3) & $61(29.2)$ \\
\hline Overall & 334 & $75(22.5)$ & 161 (48.2) & $98(29.3)$ \\
\hline
\end{tabular}

$\mathrm{p}$ for difference between male and female was 0.99 .

age (OR 1.09, 95\% CI 1.03 to 1.16), higher triglyceride (OR 1.75, 95\% CI 1.24 to 2.49), and higher $2 \mathrm{~h}$ glucose (OR 1.64, 95\% CI 1.25 to 2.16). Furthermore, current smokers carried a significantly higher risk, 3.29 times, compared with never smokers. Active physical activity showed protective effects on the outcome compared with inactive participants, with an OR of $0.34(95 \%$ CI 0.13 to 0.92 ). Results from an adjusted logistic regression analysis in females showed that the OR of baseline hypertensive individuals associated with developing diabetes was 2.38, (95\% CI 1.13 to 5.03) compared with their normotensive counterparts. Those with family history of diabetes (OR 2.74, 95\% CI 1.23 to 6.12) and higher baseline waist circumference (OR 1.05, 95\% CI 1.01 to 1.10 ) were more likely to progress to diabetes in females as well.

\section{DISCUSSION}

Diabetes has been a huge burden in China, where the incidence of diabetes has reached $11.6 \%$ and prediabetes $50.1 \%$ among adults. ${ }^{3}$ What is worse, there has been a long-running controversy about the management and treatment of prediabetes. There is evidence indicating that both, lifestyle interventions and drug treatments can substantially reduce the risk of progression to diabetes in high-risk individuals. ${ }^{4}{ }^{21}$ However, lifestyle change is difficult to implement and maintain, while for drug treatment, the benefits of prevention need to be weighed against the risk of adverse effects. ${ }^{22}$ Research is urgently needed to strengthen the evidence-based treatment guidelines for Chinese patients with prediabetes or diabetes. Our study, lasting for a decade, provides a

Table 3 Gender-related risk factors associated with regression to normal glucose regulation and the corresponding adjusted ORs

\begin{tabular}{|c|c|c|c|}
\hline Variables & Group & $\begin{array}{l}\text { Adjusted } \\
\text { OR }(95 \% \mathrm{Cl})\end{array}$ & p Value \\
\hline \multicolumn{4}{|l|}{ Male } \\
\hline Age & & \multicolumn{2}{|c|}{$0.95(0.91$ to 0.99$) 0.01$} \\
\hline Physical activity & $\begin{array}{l}\text { Inactive } \\
\text { Active }\end{array}$ & $\begin{array}{c}1 \\
3.00\end{array}(1.09$ to 8.30$)$ & 0.03 \\
\hline \multicolumn{4}{|l|}{ Female } \\
\hline Fasting glucose & & $0.24(0.08$ to 0.70$)$ & 0.009 \\
\hline 2 h Glucose & & $0.63(0.46$ to 0.86$)$ & 0.003 \\
\hline Waist circumference & & 0.94 (0.89 to 0.98$)$ & 0.005 \\
\hline
\end{tabular}

chance to observe the 10 -year outcomes of prediabetes and to explore its gender-related risk factors, thus being beneficial to the understanding of prediabetes in the general population. The major findings of our study were: (1) $22.5 \%$ participants with prediabetes had restored normal glucose regulation, $48.2 \%$ remained in a prediabetic state, and $29.3 \%$ progressed to diabetes. No gender difference was observed in the change of glucose regulation. (2) Age, $2 \mathrm{~h}$ glucose, triglycerides, smoking status, and physical activity in males, and fasting glucose, waist circumference, baseline hypertension, and family history of diabetes in females, are significantly associated with development of diabetes. (3) Physical activity in males and waist circumference in females are important factors in the restoration to normal glucose regulation in participants with prediabetes.

Our study supports the notion that, while a certain proportion of prediabetic population progresses to diabetes, the majority will stay the same or even convert back to normal glucose regulation. Previous studies showed about $5-10 \%$ of people with prediabetes progressed to diabetes every year, ${ }^{23} 24$ and $20-50 \%$ of participants returned to normal glucose regulation, ${ }^{4} 78$ although the conversion rate differs with characteristics of participants and prediabetes definitions. In an observational study investigating the natural history of diabetes in England, the proportion of participants with impaired fasting glucose at baseline who returned to normal fasting blood glucose at 10-year follow-up, was up to $55-80 \% .^{23}$ Other studies have reported lower conversion rates, for example, the Diabetes Prevention Program (DPP) outcome study reported a conversion rate of $10 \%$ in participants without any intervention. ${ }^{25}$ Our conversion rate to normal glucose regulation was in accordance with previous studies, while the progression rate to diabetes was relatively low. One of the possible reasons is the prevailing proportion of participants with isolated impaired fasting glucose in our study.

Risk factors that increase the risk of progressing to diabetes have been identified. ${ }^{12}$ However, factors delaying or reversing the pathological process remain elusive. ${ }^{13}$ The current analysis showed that while age, increased $2 \mathrm{~h}$ glucose level, triglyceride level, and current smoking, were risk factors for developing diabetes, physical activity showed its predictive value for progressing to diabetes (active vs inactive, OR 0.34, 95\% CI 0.13 to 0.92 ), as well as regressing to normal glucose regulation (active vs inactive, OR $3.00,95 \%$ CI 1.09 to 8.30 ) in males. There was evidence proving that diabetes can be prevented by changes of lifestyles in participants with prediabetes, especially physical activity. ${ }^{23}{ }^{24}$ Risk of diabetes was significantly reduced by achieving a target of $>4 \mathrm{~h}$ of exercise per week in participants who did not lose weight. What is more, the beneficial effect in preventing diabetes was similar in any type of physical activity, whether it was sports, household work or work-related physical activity. ${ }^{5}$ The China Da Qing Diabetes Prevention Outcome Study (CDQDPOS) on 
Table 4 Gender-related risk factors associated with development of diabetes and the corresponding adjusted ORs

\begin{tabular}{lllr}
\hline Variables & Group & Adjusted OR (95\%Cl) & p Value \\
\hline Male & & & \\
Age & & $1.09(1.03$ to 1.16$)$ & 0.003 \\
2 h Glucose & & $1.64(1.25$ to 2.16$)$ & $<.001$ \\
Triglycerides & Never & $1.75(1.24$ to 2.49$)$ & 0.002 \\
Smoking status & Formerly & 1 & 0.02 \\
& Currently & $3.29(0.04$ to 1.55$)$ & 1.10 to 9.78$)$ \\
Physical activity & Inactive & 1 & 0.03 \\
& Active & $0.34(0.13$ to 0.92$)$ \\
Female & & $6.09(2.47$ to 15.06) \\
Fasting glucose & & $1.05(1.01$ to 1.10$)$ \\
Waist circumference & No & 1 \\
Baseline hypertension & Yes & $2.38(1.13$ to 5.03$)$ \\
Family history of diabetes & No & 1 & 0.02 \\
& Yes & $2.74(1.23$ to 6.12$)$ \\
\hline
\end{tabular}

Chinese people with impaired glucose tolerance, provided further support to such result. It has shown that interventions targeting lifestyle changes, such as exercise and diet, produced a durable and long-lasting reduction in incidence of diabetes. Participants adopting lifestyle intervention had a $43 \%$ lower diabetes incidence for up to 14 years after the active intervention ceased, and diabetes onset was delayed on average of 3.6 years. ${ }^{26}$

Some predictors of progression to diabetes in females are modifiable, such as hypertension, fasting glucose and waist circumference. Waist circumference, a representative index of abdominal obesity, appears to be a very important factor, predicting both progression to diabetes and regression to normal glucose regulation, in our study, with every $1 \mathrm{~cm}$ increase associated with a $5 \%$ increase in risk for progression and a $6 \%$ reduction in the chance of remission. There is now a growing recognition that central rather than general obesity is more contributory to and therefore better correlates with the risk of diabetes. ${ }^{27}{ }^{28}$ Waist circumference was reported to be specifically associated with future risk of insulin resistance (IR), since it was closely associated with visceral obesity, which is a critical determinant of IR. A previous study involving 721 Mexican-Americans showed that waist circumference was a better risk predictor for diabetes than BMI was, independently of age and sex. It demonstrated a cut-off point for waist circumference $\geq 94 \mathrm{~cm}$ detected with excellent precision in those at increased risk of developing diabetes. ${ }^{29}$

Studies on restoration of prediabetes were very few to our knowledge. ${ }^{13} 30$ The DPP examined preventive strategies-intensive lifestyle modifications or metformin on the incidence of regression from prediabetes to normal glucose regulation-and found that only lifestyle modification, not metformin, is useful in achieving normoglycemia in people with prediabetes. ${ }^{13}$ However, their population was aimed at those with $\mathrm{BMI} \geq 24 \mathrm{~kg} / \mathrm{m}^{2}$ and followed only 3 years. What is more, participants with isolated impaired fasting glucose were not included in the analysis. We included a prediabetic population with any BMI and followed a much longer period, 10 years, to examine the long-term outcome in our study. Most importantly, we have explored gender-related risk factors for the regression process, which has seldom been discussed before. It will show an extensive applicability to the general population, which will further enhance the significance of our work.

Several limitations in the current study are worth noting. First, our sample size was relatively small and our response rate was relatively low. We made comparisons between the visited and unvisited participants, and found no significant difference between the two groups. However, prospective studies are still needed to confirm the current findings. Second, populations in this study were mainly participants with isolated impaired fasting glucose, and the case may have been different had analysis been aimed at individuals with impaired glucose tolerance. Finally, we did not perform a baseline HbAlc test in all participants. However, the use of HbAlc as a diagnostic test for diabetes or prediabetes was not recommended in 2003. ${ }^{31}$ It was not until 2010 that HbAlc was recognized as a diagnostic criteria of diabetes and prediabetes, ${ }^{20}$ based on which we tested HbA1c for all participants during follow-up.

In summary, active physical activity in males and lower waist circumference in females favor reversion from prediabetes to normal. In contrast, inactivity, current smoking, high $2 \mathrm{~h}$ glucose and triglyceride level in males, and high fasting glucose level, high waist circumference, and family history of diabetes and hypertension in females, significantly promote progression from prediabetes to diabetes. Intervention towards prediabetes should therefore depend on different individuals with different risk factors.

Acknowledgements The authors are grateful to the field staff and the participants of this study. 
Contributors XS designed the protocol and researched the data. MQ researched the data, analyzed the data, and prepared the manuscript. XZ researched the data. $M Q$ and $X Z$ held primary responsibility for data access. WT, HW, LJ, SS, HZ, and LG researched the data. WW contributed to the intellectual discussion. JT designed the protocol, researched the data, and reviewed the manuscript, as the guarantor of this study. All the authors read and approved of the final version of the manuscript.

Funding This study was supported by grants from the Chinese National Natural Science Foundation (81270935 and 81270860) and Transform Medicine Innovation Foundation of Shanghai Jiao Tong University School of Medicine (15ZH2001).

Competing interests None declared.

Patient consent Obtained.

Ethics approval The study protocol was approved by the institutional review board of RuiJin Hospital.

Provenance and peer review Not commissioned; externally peer reviewed.

Data sharing statement No additional data are available.

Open Access This is an Open Access article distributed in accordance with the Creative Commons Attribution Non Commercial (CC BY-NC 4.0) license, which permits others to distribute, remix, adapt, build upon this work noncommercially, and license their derivative works on different terms, provided the original work is properly cited and the use is non-commercial. See: http:// creativecommons.org/licenses/by-nc/4.0/

\section{REFERENCES}

1. Wild S, Roglic G, Green A, et al. Global prevalence of diabetes: estimates for the year 2000 and projections for 2030. Diabetes Care 2004;27:1047-53.

2. Bullard KM, Saydah SH, Imperatore G, et al. Secular changes in U. S. Prediabetes prevalence defined by hemoglobin A1c and fasting plasma glucose: National Health and Nutrition Examination Surveys, 1999-2010. Diabetes Care 2013;36:2286-93.

3. $\mathrm{Xu} \mathrm{Y,} \mathrm{Wang} \mathrm{L,} \mathrm{He} \mathrm{J,} \mathrm{et} \mathrm{al.} \mathrm{Prevalence} \mathrm{and} \mathrm{control} \mathrm{of} \mathrm{diabetes} \mathrm{in}$ Chinese adults. JAMA 2013;310:948-59.

4. Knowler WC, Barrett-Connor E, Fowler SE, et al. Reduction in the incidence of type 2 diabetes with lifestyle intervention or metformin. N Engl J Med 2002;346:393-403.

5. Tuomilehto J, Lindström J, Eriksson JG, et al. Prevention of type 2 diabetes mellitus by changes in lifestyle among participants with impaired glucose tolerance. N Engl J Med 2001;344:1343-50.

6. Torgerson JS, Hauptman J, Boldrin MN, et al. XENical in the prevention of diabetes in obese subjects (XENDOS) study: a randomized study of orlistat as an adjunct to lifestyle changes for the prevention of type 2 diabetes in obese patients. Diabetes Care 2004;27:155-61.

7. Chiasson JL, Josse RG, Gomis R, et al. Acarbose for prevention of type 2 diabetes mellitus: the STOP-NIDDM randomised trial. Lancet 2002;359:2072-7.

8. Gerstein HC, Yusuf S, Bosch J, et al. Effect of rosiglitazone on the frequency of diabetes in patients with impaired glucose tolerance or impaired fasting glucose: a randomised controlled trial. Lancet 2006;368:1096-105.

9. Legato MJ, Gelzer A, Goland R, et al. Gender-specific care of the patient with diabetes: review and recommendations. Gend Med 2006;3:131-58

10. Arnetz L, Ekberg NR, Alvarsson M. Sex differences in type 2 diabetes: focus on disease course and outcomes. Diabetes Metab Syndr Obes 2014;7:409-20.
11. Perreault L, Færch K. Approaching pre-diabetes. J Diabetes Complicat 2014;28:226-33.

12. Meigs JB, Muller DC, Nathan DM, et al. The natural history of progression from normal glucose tolerance to type 2 diabetes in the Baltimore Longitudinal Study of Aging. Diabetes 2003;52:1475-84

13. Perreault L, Kahn SE, Christophi CA, et al. Regression from pre-diabetes to normal glucose regulation in the Diabetes Prevention Program. Diabetes Care 2009;32:1583-8.

14. Tian JY, Cheng Q, Song XM, et al. Birth weight and risk of type 2 diabetes, abdominal obesity and hypertension among Chinese adults. Eur J Endocrinol 2006;155:601-7.

15. Qiu M, Shen W, Song X, et al. Effects of prediabetes mellitus alone or plus hypertension on subsequent occurrence of cardiovascular disease and diabetes mellitus: longitudinal study. Hypertension 2015;65:525-30.

16. Ainsworth BE, Haskell WL, Herrmann SD, et al. 2011 Compendium of Physical Activities: a second update of codes and MET values. Med Sci Sports Exerc 2011;43:1575-81.

17. Escobar-Jiménez F, Herrera Pombo JL, Gómez-Villalba R, et al. Standard breakfast test: an alternative to glucagon testing for C-peptide reserve? Horm Metab Res 1990;22:339-41.

18. Liao YQ. Clinical application of standard steamed bread test in diabetes diagnosis. Clin Lab J 2012;4:242-6.

19. Zhang HJ, Ji LN. Effects of oral glucose tolerance test and steamed bread test on glucose metabolism and diabetic ketoacidosis in patients with type 2 diabetes mellitus. Chin J Diab 2003;1:48-50.

20. American Diabetes Association. Diagnosis and classification of diabetes mellitus. Diabetes Care 2010;33(Suppl 1):S62-9.

21. Ramachandran A, Snehalatha C, Mary S, et al. The Indian Diabetes Prevention Programme shows that lifestyle modification and metformin prevent type 2 diabetes in Asian Indian subjects with impaired glucose tolerance (IDPP-1). Diabetologia 2006;49:289-97.

22. Del Prato S, Bianchi C, Miccoli R, et al. Pharmacological intervention in prediabetes: considering the risks and benefits. Diabetes Obes Metab 2007;9(Suppl 1):17-22.

23. Forouhi NG, Luan J, Hennings S, et al. Incidence of type 2 diabetes in England and its association with baseline impaired fasting glucose: the Ely study 1990-2000. Diabet Med 2007;24:200-7.

24. Nathan DM, Davidson MB, DeFronzo RA, et al. Impaired fasting glucose and impaired glucose tolerance: implications for care. Diabetes Care 2007;30:753-9.

25. Knowler WC, Fowler SE, Hamman RF, et al. 10-year follow-up of diabetes incidence and weight loss in the Diabetes Prevention Program Outcomes Study. Lancet 2009;374:1677-86.

26. Li G, Zhang P, Wang J, et al. The long-term effect of lifestyle interventions to prevent diabetes in the China Da Qing Diabetes Prevention Study: a 20-year follow-up study. Lancet 2008;371:1783-9.

27. Kim MK, Jang EH, Son JW, et al. Visceral obesity is a better predictor than generalized obesity for basal insulin requirement at the initiation of insulin therapy in patients with type 2 diabetes. Diabetes Res Clin Pract 2011;93:174-8.

28. Wang Y, Rimm EB, Stampfer MJ, et al. Comparison of abdominal adiposity and overall obesity in predicting risk of type 2 diabetes among men. Am J Clin Nutr 2005;81:555-63.

29. Wei M, Gaskill SP, Haffner SM, et al. Waist circumference as the best predictor of noninsulin dependent diabetes mellitus (NIDDM) compared to body mass index, waist/hip ratio and other anthropometric measurements in Mexican Americans-a 7-year prospective study. Obes Res 1997;5:16-23.

30. Dutta D, Choudhuri S, Mondal SA, et al. Urinary albumin: creatinine ratio predicts prediabetes progression to diabetes and reversal to normoglycemia: role of associated insulin resistance, inflammatory cytokines and low vitamin D. J Diabetes 2014;6:316-22.

31. Genuth $S$, Alberti KG, Bennett $P$, et al. Follow-up report on the diagnosis of diabetes mellitus. Diabetes Care 2003;26: 3160-7. 